\title{
Solutions to the Dirac Equation for Manning-Rosen Plus Shifted Deng-Fan Potential and Coulomb-Like Tensor Interaction Using Nikiforov-Uvarov Method
}

\author{
Hitler Louis ${ }^{1,2}$, Ita B. Iserom ${ }^{1}$, Magu T. Odey ${ }^{1}$, Akakuru U. Ozioma ${ }^{1}$, Nzeata-Ibe Nelson ${ }^{1}$, Ikeuba I. Alexander ${ }^{1}$, Amos I. \\ Pigweh $^{3}$, Edet C. Okon ${ }^{4}$ \\ ${ }^{1}$ Department of Pure and Applied Chemistry, Faculty of Physical Sciences, University of Calabar, Calabar, CRS, \\ Nigeria \\ ${ }^{2}$ CAS Key Laboratory for Nanosystem and Hierarchical Fabrication, CAS Centre for Excellence in Nanoscience, \\ National Centre For Nanoscience and Technology, University of Chinese Academy of Science, Beijing, China \\ ${ }^{3}$ Department of Chemistry, Faculty of Physical Sciences, Modibbo Adama University of Technology, Yola, Adamawa \\ State, Nigeria \\ ${ }^{4}$ Department of Physics, School of Physical Science, Federal University of Technology, Minna, Niger State, Nigeria \\ Correspondence: Hitler Louis, Physical/Theoretical Chemistry Research Group, Department of Pure and Applied \\ Chemistry, Faculty of Physical Sciences, University of Calabar, Calabar, CRS, Nigeria. \\ Email: louismuzong@gmail.com.
}

Received: June 11, 2018 Accepted: July 23, 2018 Online Published: August 22, 2018

doi:10.5539/ijc.v10n3p99 URL: https://doi.org/10.5539/ijc.v10n3p99

\begin{abstract}
We solve the Dirac equation for the Manning-Rosen plus shifted Deng-Fan potential including a Coulomb-like tensor potential with arbitrary spin-orbit coupling quantum number $\kappa$. In the framework of the spin and pseudospin (pspin) symmetry, we obtain the energy eigenvalue equation and the corresponding eigenfunctions in closed form by using the Nikiforov-Uvarov method. Also Special cases of the potential as been considered and their energy eigen values as well as their corresponding eigen functions are obtained for both relativistic and non-relativistic scope.
\end{abstract}

Keywords: Dirac equation, Manning-Rosen potential, shifted Deng-Fan potential, spin and pseudospin symmetry, Nikiforov-Uvarov Method

\section{Introduction}

In 1957, in an effort to find a suitable diatomic potential to describe the vibrational spectrum, Deng and Fan proposed a molecular exponential-type potential called Generalized Morse potential (Ikot et al., 2014). This potential is also known as Deng-Fan molecular potential, DF, and it is a modification of the Morse potential. This potential has been a subject of numerous studies by researchers in various applications (Oyewumi et al., 2013), and can be used to describe the mobility of nucleons in the mean field produced from the interactions of the nuclei (Maghsoodi et al., 2012). In 2012, Hamzavi et al. proposed a modified form of the DF called Shifted Deng-Fan potential, sDF (Hamzavi and Ikhdair, 2012). In the modification the DF potential shifted by dissociation energy $\alpha$. It was demonstrated in their work that sDF and Morse potential are closely similar for values of $r$ in the regions $r \approx$ re and $r>$ re however they differ at $r \approx 0$. In recent years numerous studies on the approximate analytical solution of Schrödinger equation with Manning-Rosen potential has been conducted (Awoga et al., 2013). The manning-Rosen potential was put forward to delineate the appropriate properties of diatomic molecules (Sameer Ikhdair, 2011). This potential is classified under exactly solvable and has been intensely studied. When spectrum of continuous range of values of the potential parameter could be determined analytically, such potentials are referred to as exactly solvable potentials (Min-Cang et al., 2010). Exactly solutions of wave equations with certain physical potential are of great interest in quantum mechanics. This is because the analysis makes the abstract understanding of the physical system and provides facts in affirming the impeccability of the quantum theory. This solution is paramount in examining and perfecting models and numerical methods employed for solving complex physical systems (Schiff, 1955). Recently, We reported the solution of the Dirac equation for the Manning-Rosen plus shifted Deng-Fan potential including a Yukawa-like tensor potential with arbitrary spinorbit coupling quantum number $\kappa$ where, in the framework of the spin and pseudospin (pspin) symmetry, we obtained the energy eigenvalue equation and the corresponding eigenfunctions in closed form by using the Nikiforov-Uvarov 
method. We also considered special cases of the potential and their energy eigen values as well as their corresponding eigen functions were obtained for both relativistic and non-relativistic scope (Louis et al., 2018). In this work, our aim is to solve the Dirac equation for the Manning-Rosen plus shifted Deng-Fan (MRsDF) potential in the presence of spin and pspin symmetries and by including a Coulomb-like tensor potential. The MRsDF potential takes the following form:

$$
V(r)=-\left[\frac{C e^{-\alpha r}+D e^{-2 \alpha r}}{\left(1-e^{-\alpha r}\right)^{2}}\right]+D_{e}\left[\frac{b^{2}}{\left(e^{\alpha r}-1\right)^{2}}-\frac{2 b}{\left(e^{\alpha r}-1\right)}\right] \quad \mathrm{b}=e^{\alpha r_{e}}-1,
$$

Thus eq. (1a) can be further expressed as

$$
V(r)=-\left[\frac{C e^{-\alpha r}+D e^{-2 \alpha r}}{\left(1-e^{-\alpha r}\right)^{2}}\right]+D_{e}\left[\frac{b^{2} e^{-2 \alpha r}}{\left(1-e^{-\alpha r}\right)^{2}}-\frac{2 b e^{-\alpha r}}{\left(1-e^{-\alpha r}\right)}\right]
$$

where $\alpha$ is the screening parameter, $\mathrm{C}, \mathrm{D}$ are potential depths, $D_{e}$ is the Dissociation energy, where $r_{e}$ is the equilibrium bond length.

This paper is organized as follows. In section 2, we briefly introduce the Dirac equation with scalar and vector potentials with arbitrary spin-orbit coupling quantum number $\kappa$ including tensor interaction under spin and pspin symmetry limits. The Nikiforov-Uvarov (NU) method is presented in section3.The energy eigenvalue equations and corresponding eigenfunctions are obtained in section 4. In section 5, we discussed some special cases of the potential. Finally, our conclusion is given in section 6.

\subsection{The Dirac Equation With Tensor Coupling Potential}

The Dirac equation for fermionic massive spin-1/2 particles moving in the field of an attractive scalar potential $S(r)$, a repulsive vector potential $V(r)$ and a tensor potential $U(r)$ (in units $\hbar=\mathrm{c}=1$ ) is

$$
[\vec{\alpha} \cdot \vec{p}+\beta(M+S(r))-i \beta \vec{\alpha} \cdot \vec{r} U(r)] \psi(\vec{r})=[E-V(r)] \psi(\vec{r}) .
$$

where $E$ is the relativistic binding energy of the system, $p=-i \vec{\nabla}$ is the three-dimensional momentum operator and $M$ is the mass of the fermionic particle. $\vec{\alpha}$ and $\beta$ are the $4 \times 4$ usual Dirac matrices given by

$$
\vec{\alpha}=\left(\begin{array}{cc}
0 & \vec{\sigma} \\
\vec{\sigma} & 0
\end{array}\right), \quad \beta=\left(\begin{array}{cc}
I & 0 \\
0 & -I
\end{array}\right),
$$

where $I$ is the $2 \times 2$ unitary matrix and $\vec{\sigma}$ are three-vector spin matrices

$$
\sigma_{1}=\left(\begin{array}{ll}
0 & 1 \\
1 & 0
\end{array}\right), \quad \sigma_{2}=\left(\begin{array}{cc}
0 & -i \\
i & 0
\end{array}\right), \quad \sigma_{3}=\left(\begin{array}{cc}
1 & 0 \\
0 & -1
\end{array}\right)
$$

The eigenvalues of the spin-orbit coupling operator are $\kappa=\left(j+\frac{1}{2}\right)>0$ and $\kappa=-\left(j+\frac{1}{2}\right)<0$ for unaligned spin $j=l-\frac{1}{2}$ and aligned spin $j=l+\frac{1}{2}$, respectively. The set $\left(H^{2}, K, J^{2}, J_{z}\right)$ can be taken as the complete set of conservative quantities with $\vec{J}$ being the total angular momentum operator and $K=(\vec{\sigma} \cdot \vec{L}+1)$ is the spin-orbit where $\vec{L}$ is the orbital angular momentum of the spherical nucleons that commutes with the Dirac Hamiltonian. Thus, the spinor wave functions can be classified according to their angular momentum $j$, the spin-orbit quantum number $\kappa$ and the radial quantum number $n$. Hence, they can be written as follows:

$$
\psi_{n, \kappa}(\vec{r})=\left(\begin{array}{c}
f_{n, \kappa}(\vec{r}) \\
\mathrm{g}_{n, \kappa}(\vec{r})
\end{array}\right)=\frac{1}{r}\left(\begin{array}{cc}
F_{n, \kappa}(r) & Y_{j m}^{l}(\theta, \varphi) \\
i G_{n, \kappa}(r) & Y_{j m}^{\imath}(\theta, \varphi)
\end{array}\right)
$$

where $f_{n, \kappa}(\vec{r})$ is the upper (large) component $\operatorname{andg}_{n, \kappa}(\vec{r})$ is the lower (small) component of the Dirac spinors. $Y_{j m}^{l}(\theta, \varphi)$ and $Y_{j m}^{\hat{l}}(\theta, \varphi)$ are spin and pspin spherical harmonics, respectively, and $m$ is the projection of the angular momentum on the $z$-axis. Substituting equation (5) into equation (2) and making use of the following relations

$$
\begin{gathered}
(\vec{\sigma} \cdot \vec{A})(\vec{\sigma} \cdot \vec{B})=\vec{A} \cdot \vec{B}+i \vec{\sigma} \cdot(\vec{A} \times \vec{B}), \\
(\vec{\sigma} \cdot \vec{P})=\vec{\sigma} \cdot \hat{r}\left(\hat{r} \cdot \vec{P}+i \frac{\vec{\sigma} \cdot \vec{L}}{r}\right),
\end{gathered}
$$

together with the properties 


$$
\begin{gathered}
(\vec{\sigma} \cdot \vec{L}) Y_{j m}^{\hat{l}}(\theta, \varphi)=(\kappa-1) Y_{j m}^{\hat{l}}(\theta, \varphi), \\
(\vec{\sigma} \cdot \vec{L}) Y_{j m}^{l}(\theta, \varphi)=-(\kappa-1) Y_{j m}^{l}(\theta, \varphi), \\
(\vec{\sigma} \cdot \hat{r}) Y_{j m}^{\hat{l}}(\theta, \varphi)=-Y_{j m}^{l}(\theta, \varphi), \\
(\vec{\sigma} \cdot \hat{r}) Y_{j m}^{l}(\theta, \varphi)=-Y_{j m}^{\hat{l}}(\theta, \varphi),
\end{gathered}
$$

one obtains two coupled differential equations whose solutions are the upper and lower radial wave functions $F_{n, \kappa}(r)$ and $G_{n, \kappa}(r)$ as

$$
\begin{aligned}
& \left(\frac{d}{d r}+\frac{\kappa}{r}-U(r)\right) F_{n, \kappa}(r)=\left(M+E_{n \kappa}-\Delta(r)\right) G_{n, \kappa}(r), \\
& \left(\frac{d}{d r}-\frac{\kappa}{r}+U(r)\right) G_{n, \kappa}(r)=\left(M-E_{n \kappa}+\Sigma(r)\right) F_{n, \kappa}(r),
\end{aligned}
$$

where

$$
\begin{aligned}
& \Delta(r)=V(r)-S(r), \\
& \Sigma(r)=V(r)+S(r),
\end{aligned}
$$

After eliminating $F_{n, \kappa}(r)$ and $G_{n, \kappa}(r)$ in equations (8), we obtain the following two Schrodinger-like differential equations for the upper and lower radial spinor components:

$$
\begin{gathered}
{\left[\frac{d^{2}}{d r^{2}}-\frac{\kappa(\kappa+1)}{r^{2}}+\frac{2 \kappa}{r} U(r)-\frac{d U(r)}{d r}-U^{2}(r)\right] F_{n, \kappa}(r)+\frac{\frac{d \Delta(r)}{d r}}{M+E_{n \kappa}-\Delta(r)}\left(\frac{d}{d r}+\frac{\kappa}{r}-U(r)\right) F_{n, \kappa}(r)=\left[( M + E _ { n \kappa } - \Delta ( r ) ) \left(M-E_{n \kappa}+\right.\right.} \\
\Sigma(r))] F_{n, \kappa}(r) \\
{\left[\frac{d^{2}}{d r^{2}}-\frac{\kappa(\kappa-1)}{r^{2}}+\frac{2 \kappa}{r} U(r)+\frac{d U(r)}{d r}-U^{2}(r)\right] G_{n, \kappa}(r)+\frac{\frac{d \Sigma(r)}{d r}}{M-E_{n \kappa}+\Sigma(r)}\left(\frac{d}{d r}-\frac{\kappa}{r}+U(r)\right) G_{n, \kappa}(r)=\left[( M + E _ { n \kappa } - \Delta ( r ) ) \left(M-E_{n \kappa}+\right.\right.} \\
\Sigma(r))] G_{n, \kappa}(r),
\end{gathered}
$$

respectively, where $\kappa(\kappa-1)=\hat{l}(\hat{l}+1)$ and $\kappa(\kappa+1)=l(l+1)$.

The quantum number $\kappa$ is related to the quantum numbers for spin symmetry $l$ and pspin symmetry $\hat{l}$ as

$$
\kappa=\left\{\begin{array}{c}
-(l+1)=-\left(j+\frac{1}{2}\right)\left(s_{1 / 2}, p_{3 / 2}, \text { etc }\right) \\
j=l+\frac{1}{2}, \text { aligned } \operatorname{spin}(\kappa<0), \\
+l=+\left(j+\frac{1}{2}\right)\left(p_{1 / 2}, d_{3 / 2}, \text { etc }\right) \\
j=l-\frac{1}{2}, \text { unaligned } \operatorname{spin}(\kappa>0),
\end{array}\right.
$$

and the quasidegenerate doublet structure can be expressed in terms of a pspin angular momentum $\hat{s}=1 / 2$ and pseudo-orbital angular momentum $\hat{l}$, which is defined as

$$
\kappa=\left\{\begin{array}{c}
-\hat{l}=-\left(j+\frac{1}{2}\right)\left(s_{1 / 2}, p_{3 / 2}, e t c\right) \\
j=\hat{l}-\frac{1}{2}, \text { aligned } \operatorname{spin}(\kappa<0), \\
+(\hat{l}+1)=+\left(j+\frac{1}{2}\right)\left(d_{3 / 2}, f_{5 / 2}, \text { etc }\right) \\
j=\hat{l}+\frac{1}{2}, \text { unaligned } \operatorname{spin}(\kappa>0),
\end{array}\right.
$$

where $\kappa= \pm 1, \pm 2, \ldots$. For example, $\left(1 s_{1 / 2}, 0 d_{3 / 2}\right)$ and $\left(0 p_{3 / 2}, 0 f_{5 / 2}\right)$ can be considered as pspin doublets 


\subsection{Spin Symmetry Limit}

In the spin symmetry limit, $\frac{d \Delta(r)}{d r}=0$ or $\Delta(r)=C_{s}=$ constant, with $\Sigma(r)$ taking as the MRsDF potential eq. (1b) and the coulomb-like tensor potential. i.e

$$
\begin{gathered}
\Sigma(r)=V(r)=-\left[\frac{C e^{-\alpha r}+D e^{-2 \alpha r}}{\left(1-e^{-\alpha r}\right)^{2}}\right]+D_{e}\left[\frac{b^{2} e^{-2 \alpha r}}{\left(1-e^{-\alpha r}\right)^{2}}-\frac{2 b e^{-\alpha r}}{\left(1-e^{-\alpha r}\right)}\right], \\
U(r)=-\frac{H}{r}, H=\frac{Z_{a} Z_{b} e^{2}}{4 \pi \varepsilon_{0}}, r \geq R_{c},
\end{gathered}
$$

where $R_{c}=7.78 \mathrm{fm}$ is the Coulomb radius and $\mathrm{Z}_{\mathrm{a}}$ and $\mathrm{Z}_{\mathrm{b}}$ denote the charges of the projectile a and the target nuclei $\mathrm{b}$, respectively[]. Under this symmetry, equation (10) is recast in the simple form

$$
\left[\frac{d^{2}}{d r^{2}}-\frac{\kappa(\kappa+1)}{r^{2}}-\frac{2 \kappa H}{r^{2}}-\frac{H}{r^{2}}-\frac{H^{2}}{r^{2}}\right] F_{n, \kappa}(r)=\left[\gamma\left(-\left[\frac{C e^{-\alpha r}+D e^{-2 \alpha r}}{\left(1-e^{-\alpha r}\right)^{2}}\right]+D_{e}\left[\frac{b^{2} e^{-2 \alpha r}}{\left(1-e^{-\alpha r}\right)^{2}}-\frac{2 b e^{-\alpha r}}{\left(1-e^{-\alpha r}\right)}\right]\right)+\beta^{2}\right] F_{n, \kappa}(r)
$$

where $\kappa=l$ and $\kappa=-l-1$ for $\kappa<0$ and $\kappa>0$, respectively. Also, $\gamma=\left(M+E_{n \kappa}-C_{s}\right)$ and $\beta^{2}=(M-$

$$
\left.E_{n \kappa}\right)\left(M+E_{n \kappa}-C_{s}\right) \text {. }
$$

\subsection{Pseudospin Symmetry Limit}

Lucha and Schober (2011) showed that there is a connection between pspin symmetry and near equality of the time component of a vector potential and the scalar potential, $V(r) \approx-S(r)$. After that, Ikhdair (2012) derived that if $\frac{d \Sigma(r)}{d r}=0$ or $\Sigma(r)=C_{p s}=$ constant, then pspin symmetry is exact in the Dirac equation. Here, we are taking $\Delta(r)$ as the MRsDF potential eq. (1b) and the tensor potential as the Coulomb-like potential. thus, equation (11) is recast in the simple form

$$
\left[\frac{d^{2}}{d r^{2}}-\frac{\kappa(\kappa-1)}{r^{2}}-\frac{2 \kappa H}{r^{2}}+\frac{H}{r^{2}}-\frac{H^{2}}{r^{2}}\right] G_{n, \kappa}(r)=\left[\tilde{\gamma}\left(-\left[\frac{C e^{-\alpha r}+D e^{-2 \alpha r}}{\left(1-e^{-\alpha r}\right)^{2}}\right]+D_{e}\left[\frac{b^{2} e^{-2 \propto r}}{\left(1-e^{-\alpha r}\right)^{2}}-\frac{2 b e^{-\alpha r}}{\left(1-e^{-\alpha r}\right)}\right]\right)+\tilde{\beta}^{2}\right] G_{n, \kappa}(r)
$$

where $\kappa=-\tilde{l}$ and $\kappa=\tilde{l}+1$ for $\kappa<0$ and $\kappa>0$, respectively. Also, $\tilde{\gamma}=\left(E_{n \kappa}-M-C_{p s}\right)$ and $\tilde{\beta}^{2}=(M+$ $\left.E_{n \kappa}\right)\left(M-E_{n \kappa}+C_{p s}\right)$.

to obtain the analytic solution, we use an approximation for the centrifugal term as (Louis et al., 2018) (Ita et al., 2018)

$$
\frac{1}{r^{2}}=\frac{\alpha^{2}}{\left(1-e^{-\alpha r}\right)^{2}}
$$

Finally, for the solutions to equations (16) and (17) with the above approximation, we will employ the NU method, which is briefly introduced in the following section

\section{The Nikiforov-Uvarov (NU) Method}

The NU method is based on the solutions of a generalized second order linear differential equation with special orthogonal functions. The hypergeometric (Ita et al., 2018) method has shown its power in calculating the exact energy levels of all bound states for some solvable quantum systems.

$$
\Psi_{\mathrm{n}}^{\prime \prime}(\mathrm{s})+\frac{\widetilde{\tau}(s)}{\sigma(s)} \Psi_{\mathrm{n}}^{\prime}(\mathrm{s})+\frac{\bar{\sigma}(s)}{\sigma^{2}(s)} \Psi_{\mathrm{n}}(\mathrm{s})=0
$$

Where $\sigma(\mathrm{s})$ and $\bar{\sigma}(\mathrm{s})$ are polynomials at most second degree and $\widetilde{\tau}(\mathrm{s})$ is first degree polynomials. The parametric generalization of the N-U method is given by the generalized hypergeometric-type equation

$$
\Psi^{\prime \prime}(s)+\frac{c_{1}-c_{2} s}{s\left(1-c_{3} s\right)} \Psi^{\prime}(s)+\frac{1}{s^{2}\left(1-c_{3} s\right)^{2}}\left[-\epsilon_{1} s^{2}+\epsilon_{2} s-\epsilon_{3}\right] \Psi(s)=0
$$

Thus eqn. (2) can be solved by comparing it with equation (3) and the following polynomials are obtained

$$
\widetilde{\tau}(s)=\left(c_{1}-c_{2} s\right), \sigma(s)=s\left(1-c_{3} s\right), \bar{\sigma}(s)=-\epsilon_{1} s^{2}+\epsilon_{2} s-\epsilon_{3}
$$

The parameters obtainable from equation (4) serve as important tools to finding the energy eigenvalue and eigenfunctions. They satisfy the following sets of equation respectively

$$
c_{2} n-(2 n+1) c_{5}+(2 n+1)\left(\sqrt{c_{9}}+c_{3} \sqrt{c_{8}}\right)+n(n-1) c_{3}+c_{7}+2 c_{3} c_{8}+2 \sqrt{c_{8} c_{9}}=0
$$




$$
\left(c_{2}-c_{3}\right) n+c_{3} n^{2}-(2 n+1) c_{5}+(2 n+1)\left(\sqrt{c_{9}}+c_{3} \sqrt{c_{8}}\right)+c_{7}+2 c_{3} c_{8}+2 \sqrt{c_{8} c_{9}}=0
$$

While the wave function is given as

$$
\Psi_{n}(s)=N_{n, l} S^{c_{12}}\left(1-c_{3} s\right)^{-c_{12}-\frac{c_{13}}{c_{3}}} P_{n}^{\left(c_{10}-1, \frac{c_{11}}{c_{3}}-c_{10}-1\right)}\left(1-2 c_{3} s\right)
$$

Where

$c_{4}=\frac{1}{2}\left(1-c_{1}\right), c_{5}=\frac{1}{2}\left(c_{2}-2 c_{3}\right), c_{6}=c_{5}{ }^{2}+\epsilon_{1}, c_{7}=2 c_{4} c_{5}-\epsilon_{2}, c_{8}=c_{4}{ }^{2}+\epsilon_{3}$,

$c_{9}=c_{3} c_{7}+c_{3}^{2} c_{8}+c_{6}, c_{10}=c_{1}+2 c_{4}+2 \sqrt{c_{8}}, c_{11}=c_{2}-2 c_{5}+2\left(\sqrt{c_{9}}+\mathrm{c} 3 \sqrt{c_{8}}\right)$

$c_{12}=c_{4}+\sqrt{c_{8}}, c_{13}=c_{5}-\left(\sqrt{c_{9}}+\mathrm{c} 3 \sqrt{c_{8}}\right)$

and $P_{n}$ is the orthogonal polynomials.

\section{Solutions to the Dirac Equation}

We will now solve the Dirac equation with the MRsDF potential and tensor potential by using the NU method.

\subsection{The Spin Symmetric Case}

To obtain the solution to equation (16), by using the transformation $s=e^{-\alpha r}$, we rewrite it as follows:

$\frac{d^{2} F_{n, \kappa}(s)}{d s^{2}}+\frac{(1-s)}{s(1-s)} \frac{d F_{n, \kappa}(s)}{d s}+\frac{1}{s^{2}(1-s)^{2}}\left[-\eta_{\kappa}\left(\eta_{\kappa}-1\right)+\frac{\gamma}{\alpha^{2}}\left(C s+D s^{2}-D_{e} b^{2} s^{2}+2 D_{e} b s(1-s)\right)-\frac{\beta^{2}}{\alpha^{2}}(1-s)^{2}\right] F_{n, \kappa}(s)=0$,

Eq. (26) is further simplified as

$\frac{d^{2} F_{n, \kappa}(s)}{d s^{2}}+\frac{(1-s)}{s(1-s)} \frac{d F_{n, \kappa}(s)}{d s}+\frac{1}{s^{2}(1-s)^{2}}\left[-\left(\frac{\beta^{2}}{\alpha^{2}}-\frac{2 \gamma D_{e} b}{\alpha^{2}}+\frac{\gamma D_{e} b^{2}}{\alpha^{2}}-\frac{\gamma D}{\alpha^{2}}\right) s^{2}+\left(\frac{2 \beta^{2}}{\alpha^{2}}+\frac{2 \gamma D_{e} b}{\alpha^{2}}+\frac{\gamma C}{\alpha^{2}}\right) s-\left(\frac{\beta^{2}}{\alpha^{2}}+\eta_{\kappa}\left(\eta_{\kappa}-1\right)\right)\right] F_{n, \kappa}(s)=0$,

where $\eta_{\kappa}=\kappa+H+1$, Comparing eq. (27) with eq. (20), we obtain

$$
\begin{aligned}
& c_{1}=1, \quad \epsilon_{1}=\frac{\beta^{2}}{\alpha^{2}}-\frac{2 \gamma D_{e} b}{\alpha^{2}}+\frac{\gamma D_{e} b^{2}}{\alpha^{2}}-\frac{\gamma D}{\alpha^{2}} \\
& c_{2}=1, \quad \epsilon_{2}=\frac{2 \beta^{2}}{\alpha^{2}}+\frac{2 \gamma D_{e} b}{\alpha^{2}}+\frac{\gamma C}{\alpha^{2}} \\
& c_{3}=1, \quad \epsilon_{3}=\frac{\beta^{2}}{\alpha^{2}}+\eta_{\kappa}\left(\eta_{\kappa}-1\right)
\end{aligned}
$$

and from eq. (25), we further obtain

$$
\begin{aligned}
& c_{4}=0, \quad c_{5}=-\frac{1}{2}, \\
& c_{6}=\frac{1}{4}+\frac{\beta^{2}}{\alpha^{2}}-\frac{2 \gamma D_{e} b}{\alpha^{2}}+\frac{\gamma D_{e} b^{2}}{\alpha^{2}}-\frac{\gamma D}{\alpha^{2}}, \quad c_{7}=-\left(\frac{2 \beta^{2}}{\alpha^{2}}+\frac{2 \gamma D_{e} b}{\alpha^{2}}+\frac{\gamma C}{\alpha^{2}}\right), \\
& c_{8}=\frac{\beta^{2}}{\alpha^{2}}+\eta_{\kappa}\left(\eta_{\kappa}-1\right), \quad c_{9}=\left(\eta_{\kappa}-\frac{1}{2}\right)^{2}-\frac{\gamma D}{\alpha^{2}}-\frac{\gamma C}{\alpha^{2}}+\frac{\gamma D_{e} b^{2}}{\alpha^{2}}, \\
& c_{10}=1+2 \sqrt{\frac{\beta^{2}}{\alpha^{2}}+\eta_{\kappa}\left(\eta_{\kappa}-1\right)}, \\
& c_{11}=2+2\left(\sqrt{\left(\eta_{\kappa}-\frac{1}{2}\right)^{2}-\frac{\gamma D}{\alpha^{2}}-\frac{\gamma C}{\alpha^{2}}+\frac{\gamma D_{D} b^{2}}{\alpha^{2}}}+\sqrt{\frac{\beta^{2}}{\alpha^{2}}+\eta_{\kappa}\left(\eta_{\kappa}-1\right)}\right), \\
& c_{12}=\sqrt{\frac{\beta^{2}}{\alpha^{2}}+\eta_{\kappa}\left(\eta_{\kappa}-1\right)}, \\
& c_{13}=-\frac{1}{2}-\left(\sqrt{\left(\eta_{\kappa}-\frac{1}{2}\right)^{2}-\frac{\gamma D}{\alpha^{2}}-\frac{\gamma C}{\alpha^{2}}+\frac{\gamma D_{e} b^{2}}{\alpha^{2}}}+\sqrt{\frac{\beta^{2}}{\alpha^{2}}+\eta_{\kappa}\left(\eta_{\kappa}-1\right)}\right)
\end{aligned}
$$

In addition, the energy eigenvalue equation can be obtained by using eq. (23) as follows: 


$$
\left(n+\frac{1}{2}+\sqrt{\left(\eta_{\kappa}-\frac{1}{2}\right)^{2}-\frac{\gamma D}{\alpha^{2}}-\frac{\gamma C}{\alpha^{2}}+\frac{\gamma D_{e} b^{2}}{\alpha^{2}}}+\sqrt{\frac{\beta^{2}}{\alpha^{2}}+\eta_{\kappa}\left(\eta_{\kappa}-1\right)}\right)^{2}=\frac{\beta^{2}}{\alpha^{2}}-\frac{\gamma D}{\alpha^{2}}+\frac{2 \gamma D_{e} b}{\alpha^{2}}+\frac{\gamma D_{e} b^{2}}{\alpha^{2}}
$$

By substituting the explicit forms of $\gamma$ and $\beta^{2}$ after equation (16) into equation (30), one can readily obtain the closed form for the energy formula.

$$
\begin{gathered}
\left(n+\frac{1}{2}+\sqrt{\left(\eta_{\kappa}-\frac{1}{2}\right)^{2}-\frac{D}{\alpha^{2}}\left(M+E_{n \kappa}-C_{s}\right)-\frac{C}{\alpha^{2}}\left(M+E_{n \kappa}-C_{s}\right)+\frac{D_{e} b^{2}}{\alpha^{2}}\left(M+E_{n \kappa}-C_{s}\right)+}\right. \\
\left.\sqrt{\frac{1}{\alpha^{2}}\left(\left(M-E_{n \kappa}\right)\left(M+E_{n \kappa}-C_{s}\right)\right)+\eta_{\kappa}\left(\eta_{\kappa}-1\right)}\right)^{2}=\frac{1}{\alpha^{2}}\left(\left(M-E_{n \kappa}\right)\left(M+E_{n \kappa}-C_{s}\right)\right)-\frac{D}{\alpha^{2}}\left(M+E_{n \kappa}-C_{s}\right)+\frac{D_{e} b^{2}}{\alpha^{2}}\left(M+E_{n \kappa}-\right. \\
\left.C_{s}\right)+\frac{2 D_{e} b}{\alpha^{2}}\left(M+E_{n \kappa}-C_{s}\right)
\end{gathered}
$$

On the other hand, to find the corresponding wave functions, referring to equation (29) and eq. (24), we obtain the upper component of the Dirac spinor from eq. 24 as

$$
F_{n, \kappa}(s)=B_{n, \kappa} S \sqrt{\frac{\beta^{2}}{\alpha^{2}}+\eta_{\kappa}\left(\eta_{\kappa}-1\right)}(1-s)^{\frac{1}{2}+\sqrt{\left(\eta_{\kappa}-\frac{1}{2}\right)^{2}-\frac{\gamma D}{\alpha^{2}}-\frac{\gamma C}{\alpha^{2}}+\frac{\gamma D e b^{2}}{\alpha^{2}}}} P_{n}\left(2 \sqrt{\frac{\beta^{2}}{\alpha^{2}}+\eta_{\kappa}\left(\eta_{\kappa}-1\right), 2} \sqrt{\left(\eta_{\kappa}-\frac{1}{2}\right)^{2}-\frac{\gamma D}{\alpha^{2}}-\frac{\gamma C}{\alpha^{2}}+\frac{\gamma D D_{e} b^{2}}{\alpha^{2}}}\right)(1-2 s)
$$

where $B_{n, \kappa}$ is the normalization constant. The lower component of the Dirac spinor can be calculated from equation (8a)

$$
G_{n, \kappa}(r)=\frac{1}{\left(M+E_{n \kappa}-C_{S}\right)}\left(\frac{d}{d r}+\frac{\kappa}{r}-U(r)\right) F_{n, \kappa}(r)
$$

where $E_{n \kappa} \neq-M+C_{s}$.

\subsection{The Pseudospin Symmetric Case}

To avoid repetition in the solution of equation (17), we follow the same procedures explained in section 4.1 and hence obtain the following energy eigenvalue equation:

$$
\left(n+\frac{1}{2}+\sqrt{\left(\Lambda_{\kappa}-\frac{1}{2}\right)^{2}-\frac{\tilde{\gamma} D}{\alpha^{2}}-\frac{\tilde{\gamma} C}{\alpha^{2}}+\frac{\widetilde{\gamma} D_{e} b^{2}}{\alpha^{2}}}+\sqrt{\frac{\widetilde{\beta}^{2}}{\alpha^{2}}+\Lambda_{\kappa}\left(\Lambda_{\kappa}-1\right)}\right)^{2}=\frac{\widetilde{\beta}^{2}}{\alpha^{2}}-\frac{\widetilde{\gamma} D}{\alpha^{2}}+\frac{\widetilde{\gamma} D_{e} b^{2}}{\alpha^{2}}+\frac{2 \widetilde{\gamma} D_{e} b}{\alpha^{2}}
$$

By substituting the explicit forms of $\tilde{\gamma}$ and $\tilde{\beta}^{2}$ after equation (17b) into equation (34), one can readily obtain the closed form for the energy formula as

$$
\begin{gathered}
\left(n+\frac{1}{2}+\sqrt{\left(\Lambda_{\kappa}-\frac{1}{2}\right)^{2}-\frac{D}{\alpha^{2}}\left(E_{n \kappa}-M-C_{p s}\right)-\frac{C}{\alpha^{2}}\left(E_{n \kappa}-M-C_{p s}\right)+\frac{D_{e} b^{2}}{\alpha^{2}}\left(E_{n \kappa}-M-C_{p s}\right)}+\right. \\
\left.\sqrt{\frac{1}{\alpha^{2}}\left(\left(M+E_{n \kappa}\right)\left(M-E_{n \kappa}+C_{p s}\right)\right)+\Lambda_{\kappa}\left(\Lambda_{\kappa}-1\right)}\right)^{2}=\frac{1}{\alpha^{2}}\left(\left(M+E_{n \kappa}\right)\left(M-E_{n \kappa}+C_{s}\right)\right)-\frac{D}{\alpha^{2}}\left(E_{n \kappa}-M-C_{p s}\right)+\frac{D_{e} b^{2}}{\alpha^{2}}\left(E_{n \kappa}-M-\right. \\
\left.C_{p s}\right)+\frac{2 D_{e} b}{\alpha^{2}}\left(E_{n \kappa}-M-C_{p s}\right)
\end{gathered}
$$

and the corresponding wave functions for the upper Dirac spinor as

$$
G_{n, \kappa}(r)=\tilde{B}_{n, \kappa} S^{\sqrt{\frac{\tilde{\beta}^{2}}{\alpha^{2}}+\Lambda_{\kappa}\left(\Lambda_{\kappa}-1\right)}}(1-s)^{\frac{1}{2}+\sqrt{\left(\Lambda_{\kappa}-\frac{1}{2}\right)^{2}-\frac{\tilde{\gamma} D}{\alpha^{2}}-\frac{\tilde{\gamma} C}{\alpha^{2}}+\frac{\tilde{\gamma} D_{e} b^{2}}{\alpha^{2}}} P_{n}\left(2 \sqrt{\frac{\tilde{\beta}^{2}}{\alpha^{2}}+\Lambda_{\kappa}\left(\Lambda_{\kappa}-1\right), 2} \sqrt{\left(\Lambda_{\kappa}-\frac{1}{2}\right)^{2}-\frac{\tilde{\gamma} D}{\alpha^{2}}-\frac{\tilde{\gamma} C}{\alpha^{2}}+\frac{\tilde{\gamma} D_{e} b^{2}}{\alpha^{2}}}\right)}(1-2 s)
$$

were $\Lambda_{\kappa}=\kappa+H$ and $\tilde{B}_{n, \kappa}$ is the normalization constant. Finally, the Upper-spinor component of the Dirac equation can be obtained via equation $(8 \mathrm{~b})$ as

$$
F_{n, \kappa}(r)=\frac{1}{\left(M-E_{n \kappa}+C_{p s}\right)}\left(\frac{d}{d r}-\frac{\kappa}{r}+U(r)\right) G_{n, \kappa}(r)
$$

where $E_{n \kappa} \neq M+C_{p s}$.

\section{Discussion}

In this section, we are going to study some special cases of the energy eigenvalues given by Eqs. (31) and (35) for the spin and pseudospin symmetries, respectively.

Case 1. If one sets $C_{s}=0, C_{p s}=0, D_{e}=0$ in eq. (31) and eq. (35), we obtain the energy equation of Manning-Rosen potential for spin and pseudospin symmetric Dirac theory respectively, 


$$
\begin{gathered}
\left(n+\frac{1}{2}+\sqrt{\left(\eta_{\kappa}-\frac{1}{2}\right)^{2}-\frac{D}{\alpha^{2}}\left(M+E_{n \kappa}\right)-\frac{C}{\alpha^{2}}\left(M+E_{n \kappa}\right)}+\sqrt{\frac{1}{\alpha^{2}}\left(\left(M-E_{n \kappa}\right)\left(M+E_{n \kappa}\right)\right)+\eta_{\kappa}\left(\eta_{\kappa}-1\right)}\right)^{2}=\frac{1}{\alpha^{2}}\left(\left(M-E_{n \kappa}\right)(M+\right. \\
\left.\left.E_{n \kappa}\right)\right)-\frac{D}{\alpha^{2}}\left(M+E_{n \kappa}\right)
\end{gathered}
$$

and

$$
\begin{gathered}
\left(n+\frac{1}{2}+\sqrt{\left(\Lambda_{\kappa}-\frac{1}{2}\right)^{2}-\frac{D}{\alpha^{2}}\left(E_{n \kappa}-M\right)-\frac{C}{\alpha^{2}}\left(E_{n \kappa}-M\right)}+\sqrt{\frac{1}{\alpha^{2}}\left(M+E_{n \kappa}\right)\left(M-E_{n \kappa}\right)+\Lambda_{\kappa}\left(\Lambda_{\kappa}-1\right)}\right)^{2}=\frac{1}{\alpha^{2}}\left(M+E_{n \kappa}\right)\left(M-E_{n \kappa}\right)- \\
\frac{D}{\alpha^{2}}\left(E_{n \kappa}-M\right)
\end{gathered}
$$

Case 2: If one sets $C_{s}=0, C_{p s}=0, C=D=0$ in eq. (31) and eq. (35), we obtain the energy equation of shifted Deng-Fan potential for spin and pseudospin symmetric Dirac theory respectively,

$$
\begin{gathered}
\left(n+\frac{1}{2}+\sqrt{\left(\eta_{\kappa}-\frac{1}{2}\right)^{2}+\frac{D_{e} b^{2}}{\alpha^{2}}\left(M+E_{n \kappa}-C_{s}\right)}+\sqrt{\frac{1}{\alpha^{2}}\left(\left(M-E_{n \kappa}\right)\left(M+E_{n \kappa}-C_{s}\right)\right)+\eta_{\kappa}\left(\eta_{\kappa}-1\right)}\right)^{2}=\frac{1}{\alpha^{2}}\left(( M - E _ { n \kappa } ) \left(M+E_{n \kappa}-\right.\right. \\
\left.\left.C_{s}\right)\right)+\frac{D_{e} b^{2}}{\alpha^{2}}\left(M+E_{n \kappa}-C_{s}\right)+\frac{2 D_{e} b}{\alpha^{2}}\left(M+E_{n \kappa}-C_{s}\right)
\end{gathered}
$$

and

$$
\begin{gathered}
\left(n+\frac{1}{2}+\sqrt{\left(\Lambda_{\kappa}-\frac{1}{2}\right)^{2}+\frac{D_{e} b^{2}}{\alpha^{2}}\left(E_{n \kappa}-M-C_{p s}\right)}+\sqrt{\frac{1}{\alpha^{2}}\left(\left(M+E_{n \kappa}\right)\left(M-E_{n \kappa}+C_{p s}\right)\right)+\Lambda_{\kappa}\left(\Lambda_{\kappa}-1\right)}\right)^{2}=\frac{1}{\alpha^{2}}\left(( M + E _ { n \kappa } ) \left(M-E_{n \kappa}+\right.\right. \\
\left.\left.C_{S}\right)\right)-\frac{D}{\alpha^{2}}\left(E_{n \kappa}-M-C_{p s}\right)+\frac{D_{e} b^{2}}{\alpha^{2}}\left(E_{n \kappa}-M-C_{p s}\right)+\frac{2 D_{e} b}{\alpha^{2}}\left(E_{n \kappa}-M-C_{p s}\right)
\end{gathered}
$$

Case 3: Let us now discuss the relativistic limit of the energy eigenvalues and wavefunctions of our solutions. If we take $C_{s}=0, H=0, \kappa \rightarrow l$ and put $S(r)=V(r)=\Sigma(r)$, the nonrelativistic limit of energy equation 31 for MRsDF potential and wave function 32 under the following appropriate transformations $M+E_{n \kappa} \rightarrow \frac{2 \mu}{\hbar^{2}}$, and $M-E_{n \kappa} \rightarrow$ $-E_{n l}$ becomes

$$
E_{n l}=-\frac{\alpha^{2} \hbar^{2}}{2 \mu}\left\{\left[\frac{2 l(l+1)-\frac{2 \mu C}{\alpha^{2} \hbar^{2}}+\frac{4 \mu D e}{\alpha^{2} \hbar^{2}} b+\left(n^{2}+n+\frac{1}{2}\right)+(2 n+1) \sqrt{\left(l+\frac{1}{2}\right)^{2}-\frac{2 \mu C}{\alpha^{2} \hbar^{2}}-\frac{2 \mu D}{\alpha^{2} \hbar^{2}}+\frac{2 \mu D e}{\alpha^{2} \hbar^{2}} b^{2}}}{(2 n+1)+2 \sqrt{\left(l+\frac{1}{2}\right)^{2}-\frac{2 \mu C}{\alpha^{2} \hbar^{2}}-\frac{2 \mu D}{\alpha^{2} \hbar^{2}}+\frac{2 \mu D e}{\alpha^{2} \hbar^{2}} b^{2}}}\right]^{2}-l(l+1)\right\}
$$

and the associated wave functions $F_{n \kappa}(s) \rightarrow R_{n, l}(s)$ are

$$
\begin{gathered}
R_{n, l}(s)=N_{n, l} S^{U / 2}(1-s)^{(V-1) / 2} P_{n}^{(U, V)}(1-2 s), \\
\text { where } U=2 \sqrt{\frac{2 \mu E_{n l}}{\alpha^{2} \hbar^{2}}+l(l+1)} \text { and } V=2 \sqrt{\left(l+\frac{1}{2}\right)^{2}-\frac{2 \mu C}{\alpha^{2} \hbar^{2}}-\frac{2 \mu D}{\alpha^{2} \hbar^{2}}+\frac{2 \mu D_{e}}{\alpha^{2} \hbar^{2}} b^{2}}
\end{gathered}
$$

Case 4: If $D_{e}=0$ in eq. (42), we obtain the energy equation of Manning-Rosen potential in the non-relativistic limit

$$
E_{n l}=-\frac{\alpha^{2} \hbar^{2}}{2 \mu}\left\{\left[\frac{2 l(l+1)-\frac{2 \mu C}{\alpha^{2} \hbar^{2}}+\left(n^{2}+n+\frac{1}{2}\right)+(2 n+1) \sqrt{\left(l+\frac{1}{2}\right)^{2}-\frac{2 \mu C}{\alpha^{2} \hbar^{2}}-\frac{2 \mu D}{\alpha^{2} \hbar^{2}}}}{(2 n+1)+2 \sqrt{\left(l+\frac{1}{2}\right)^{2}-\frac{2 \mu C}{\alpha^{2} \hbar^{2}}-\frac{2 \mu D}{\alpha^{2} \hbar^{2}}}}\right]^{2}-l(l+1)\right\}
$$

Case 5: If $C=D=0$ in eq. (42), we obtain the energy equation of the shifted Deng-Fan potential in the non-relativistic limit

$$
E_{n l}=-\frac{\alpha^{2} \hbar^{2}}{2 \mu}\left\{\left[\frac{2 l(l+1)+\frac{4 \mu D_{e}}{\alpha^{2} \hbar^{2}} b+\left(n^{2}+n+\frac{1}{2}\right)+(2 n+1) \sqrt{\left(l+\frac{1}{2}\right)^{2}+\frac{2 \mu D e}{\alpha^{2} \hbar^{2}} b^{2}}}{(2 n+1)+2 \sqrt{\left(l+\frac{1}{2}\right)^{2}+\frac{2 \mu D e}{\alpha^{2} \hbar^{2}} b^{2}}}\right]^{2}-l(l+1)\right\}
$$

\section{Conclusion}

In this work, using the parametric generalization of the NU method, we have obtained approximately energy eigenvalues and the corresponding wave functions of the Dirac equation for the Manning-Rosen plus shifted Deng-Fan potential including a Coulomb-like tensor potential with arbitrary spin-orbit coupling quantum number $\kappa$. The corresponding unnormalized eigen functions are evaluated in terms of Jacobi polynomials. Interestingly, the 
Klein-Gordon and Dirac equation with the arbitrary angular momentum values for this potential can be solved by this method. The resulting eigen energy equations can be used to study the spectroscopy of some selected diatomic atoms and molecules.

\section{Acknowledgements}

We thank our colleagues in the Department of Mathematics for their insight and useful suggestions

\section{References}

Awoga, O. A., Ikot, A. N., \& Emah, J. B. (2013). Bound state solutions of deformed generalized Deng-Fan potential plus deformed Eckart potential in D-dimensions. Revista mexicana de física, 59(3), 229-235.

Hamzavi, M., Ikhdair, S. M., \& Thylwe, K. E. (2013). Equivalence of the empirical shifted Deng-Fan oscillator potential for diatomic molecules. Journal of Mathematical Chemistry, 51(1), 227-238. https://doi.org/10.1007/s10910-012-0075-x

Ikhdair, S. M. (2011). On the bound-state solutions of the Manning-Rosen potential including an improved approximation to the orbital centrifugal term. Physica Scripta,83(1), 015010. https://doi.org/10.1088/0031-8949/83/01/015010

Ikot, A. N., Hassanabadi, H., Yazarloo, B. H., Umo, M. I., \& Zarrinkamar, S. (2014). Dirac-Deng-Fan Problem with Coulomb-Hulthen Tensor Interactions. Acta Phys. Polonica A, 126, 656. https://doi.org/10.12693/APhysPolA.126.656

Ita, B. I., Nzeata-Ibe, N., Magu, T. O., \& Hitler, L. (2018). Bound-State Solutions of the Schrödinger Equation with Woods-Saxon Plus Attractive Inversely Quadratic Potential via Parametric Nikiforov-Uvarov Method.

Louis, H., Iserom, I. B., Akakuru, O. U., Nzeata-Ibe, N. A., Ikeuba, A. I., Magu, T. O., ... \& Collins, E. O. (2018). L-state Solutions of the Relativistic and Non-Relativistic Wave Equations for Modified Hylleraas-Hulthen Potential Using the Nikiforov-Uvarov Quantum Formalism. Oriental Journal of physical Sciences, 3(1).

Louis, H., Ita, B. I., Amos, P. I., Akakuru, O. U., Orosun, M. M., Nzeata-Ibe, N. A., \& Philip, M. (2018). Bound State Solutions of Klein-Gordon Equation with Manning-Rosen Plus a Class of Yukawa Potential Using Pekeris-Like Approximation of the Coulomb Term and Parametric Nikiforov-Uvarov, IJCPS, 7(1). https://doi.org/10.30731/ijcps.7.1.2018.33-37

Lucha, W., \& Schöberl, F. F. (1999). Solving the Schrödinger equation for bound states with Mathematica 3.0. International Journal of Modern Physics C, 10(04), 607-619. https://doi.org/10.1142/S0129183199000450

Maghsoodi, E., Hassanabadi, H., \& Zarrinkamar, S. (2012). Spectrum of dirac equation under Deng-Fan scalar and vector potentials and a Coulomb tensor interaction by SUSYQM. Few-Body Systems, 53(3-4), 525-538. https://doi.org/10.1007/s00601-012-0314-5

Min-Cang, Z., \& Bo, A. (2010). Analytical Solutions of the Manning-Rosen Potential In the Tridiagonal Program. Chinese Physics Letters, 27(11), 110301. https://doi.org/10.1088/0256-307X/27/11/110301

Oyewumi, K. J., Oluwadare, O. J., Sen, K. D., \& Babalola, O. A. (2013). Bound state solutions of the Deng-Fan molecular potential with the Pekeris-type approximation using the Nikiforov-Uvarov $(\mathrm{N}-\mathrm{U})$ method. Journal of Mathematical Chemistry, 51(3), 976-991. https://doi.org/10.1007/s10910-012-0123-6

Schiff, L. I. (1959). Gravitational properties of antimatter. Proceedings of the National Academy of Sciences, 45(1), 69-80. https://doi.org/10.1073/pnas.45.1.69

\section{Copyrights}

Copyright for this article is retained by the author(s), with first publication rights granted to the journal.

This is an open-access article distributed under the terms and conditions of the Creative Commons Attribution license (http://creativecommons.org/licenses/by/4.0/). 